\title{
Evaluación del impacto socioeconómico del microcrédito en la zona oriental de la provincia del Azuay
}

Juan Moscoso Calle/Diana Calle Ramírez*

\begin{abstract}
Resumen
La presente investigación está ligada a un estudio sobre el impacto del microcrédito en la economía de la zona oriental de la provincia del Azuay, en el que se consideran factores y variables cuantitativas que permitan establecer información más consistente a través de diferentes modelos que nos retroalimenten sobre la eficiencia y eficacia de la entrega de esos recursos crediticios. Los diagnósticos realizados, las variables estudiadas y los modelos propuestos son producto de un análisis y/o estudio económico y estadístico que se realizó en un trabajo de campo con las instituciones financieras de la zona y los beneficiarios del microcrédito.
\end{abstract}

\section{Palabras clave}

Impacto microcrédito, diagnostico microcrédito Azuay, análisis microcrédito

\begin{abstract}
This research is linked to a study on the impact of microcredit on the economy of the eastern province of Azuay, in which variables are considered factors and quantitative information to establish more consistent across different models that provide feedback on efficiency and effectiveness of delivery of those resources credit. The studies carried out, the variables studied and proposed models are the result of an analysis and / or economic and statistical study was conducted in a field with area financial institutions and the beneficiaries of microcredit.
\end{abstract}

\section{Key words}

Impact Microcredit, microcredit diagnosis Azuay, micro analysis

Forma sugerida de citar: Moscoso Calle, Juan; Calle, Diana. 2011. Evaluación del impacto socioeconómico del microcrédito en la zona oriental de la provincia del Azuay. Retos 1. Enero/Junio. Pp. 5-12

\footnotetext{
* Docentes de la Universidad Politécnica Salesiana. Carrera de Administración de Empresas Sede Cuenca. moscoso@ups.edu.ec/dcalle@ups.edu.ec.
} 
La Evaluación de Impacto generalmente ha estado relacionada con factores cualitativos[1] antes que fundamentada en elementos cuantitativos, que miden características o variables que pueden tomar valores numéricos y cuyos resultados se obtienen mediante un análisis estadístico. La razón es que la descripción o investigación de situaciones observables es más fácil que extraer información cuantitativa sobre ciertas variables. En el caso del microcrédito, la observación o pequeña investigación (cuantitativa) ha evidenciado que este ha sido una herramienta de financiamiento para las clases económicas bajas, en su mayoría a través de un simple conteo estadístico histórico, en que los hitos identifican las situaciones antes y después de fenómenos de cualquier índole; en el caso de la zona oriental de la provincia del Azuay, estos hitos han sido el Desastre de la Josefina y la Crisis económica de 1999.

Para responder la pregunta de cómo esos recursos entregados en el microcrédito han retroalimentado a los ingresos, capital de trabajo, apalancamiento para otras actividades, etcétera, es necesario un estudio más profundo en donde las variables identificadas estarían expresadas en números, y serían el resultado de comparar las situaciones antes y después de los hitos anteriormente indicados.

Desde el punto de vista económico, las situaciones de antes y después se fundamentan en la teoría de Evaluación de Proyectos (Sapag, 2000) en la que se describe una situación (costos y beneficios) antes de hacer una inversión y se describe la situación (costos y beneficios) después de invertir, operar y evaluar, verificando si ha convenido o no hacer esa inversión. Esta particularidad se ajusta a nuestra investigación, ya que la situación sería el microcrédito y los costos o beneficios serían las variables económicas y financieras de las familias que accedieron a ese financiamiento.

Las preguntas a responder: ¿En qué y cuánto ha impactado el microcrédito en las economías familiares de la zona oriental de la provincia del Azuay[2]? ¿Ha sido el microcrédito un elemento que ha favorecido al desarrollo de la región?

Para afrontar el tema cuantitativo de la evaluación de impacto del microcrédito, partimos de una situación antes (ex ante) y una situación después (ex pos), tal como se demuestra en el gráfico siguiente:

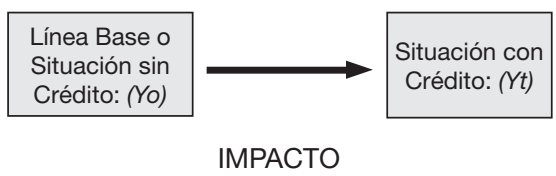

Inicialmente, podemos observar que el cambio en cualquier variable (Y), antes y después del crédito, se podría aceptar como una medida de impacto del microcrédito en las condiciones de los beneficiarios (microempresarios), representados por esa variable. Este 
cálculo se puede representar en la siguiente ecuación: $\mathrm{K}=\mathrm{Y}_{\mathrm{t}}-\mathrm{Y}_{\mathrm{O}}=\Delta \mathrm{Y}$ donde $\mathrm{K}$ es el impacto, Yo, es la variable de resultado antes de obtener el crédito y Yt es la situación ex pos.

Para nuestra evaluación de impacto del microcrédito, es importante indicar que estas variables no pueden ser consideradas como únicas, ya que $\mathrm{K}$ puede estar influenciada por varios factores o múltiples variables, pues si no consideramos a todos se puede incurrir en una sobreestimación o subestimación del verdadero impacto generado por el microcrédito.

\section{Materiales y métodos}

El levantamiento de la información tuvo sus particularidades, ya que necesitábamos establecer la variable de impacto antes de obtener el microcrédito (Yo), pues no se levantó una Línea Base en su momento; es decir, uno o varios años atrás. Ante esta situación, el criterio fue recurrir a las carpetas que presentan los microempresarios al solicitar un crédito, con la información económica y financiera, que corresponde a la Línea Base necesaria para nuestros fines. En cada una de estas carpetas se encuentran las diferentes variables de estudio, resumidas en la Ficha de solicitud de crédito[3]. Con base en este documento procedimos a estructurar una matriz para levantar la información requerida[4].

Retos I(I): 20II

(C) 2011, Universidad Politécnica Salesiana, Ecuador
Luego de la Línea Base, era necesario actualizar la información (ex pos), para lo que usamos la misma matriz pero con información a la fecha; así podemos contrastar el antes con el después, pero en función de las mismas variables, por ejemplo: "antes del crédito el microempresario disponía de $\$ 100.000,00$ de patrimonio, en la actualidad dispone de $\$ 120.000,00 ”$. Se fue registrando toda esta información en la ficha respectiva. La información ex ante y ex pos fue procesada en programas estadísticos, que facilitan las pruebas que se requieran. En este sentido, se utilizó la prueba de Wilcoxon[5] para contrastar las diferencias positivas y negativas entre los valores de las variables Yt y Yo.

Por la construcción de las variables y la tipología de la información procesada no se realizaron supuestos sobre la distribución de las variables, sino un test no paramétrico[6], que considera a un microcrédito como un tratamiento y prueba estadísticamente su efecto en variables consideradas como beneficios: Capital de Trabajo, Flujos de Caja e Ingresos. Su análisis determinó a estas tres categorías como las de mayor incidencia, que harían posible encontrar diferencias notables en el momento de procesar los datos.

\section{Resultados}

Los modelos de impacto socioeconómico del microcrédito 
están expresados en dos líneas de acción: 1. Establecen ciertas pautas de gestión para los actores que ofertan este tipo de serviciosrecursos, con ciertas estrategias de acción que inciden en el mejoramiento de la cartera y el alcance del microcrédito en los diferentes estratos económicos de la población en estudio. 2. Por medio del diagnóstico establecido, se definen algunas variables que permiten determinar el contraste entre lo positivo y lo negativo del mi- crocrédito en éstas. Los resultados obtenidos son los siguientes:

\section{Recopilación de datos e in- formación para diagnóstico y levantamiento de información ex ante y ex pos}

El levantamiento de esta información en las instituciones financieras y en los hogares de los clientes con crédito fue hecha con el apoyo de estudiantes de 5 to y $7 \mathrm{mo}$ ciclo de la carrera de Administración de Empresas de la Universidad Politécnica

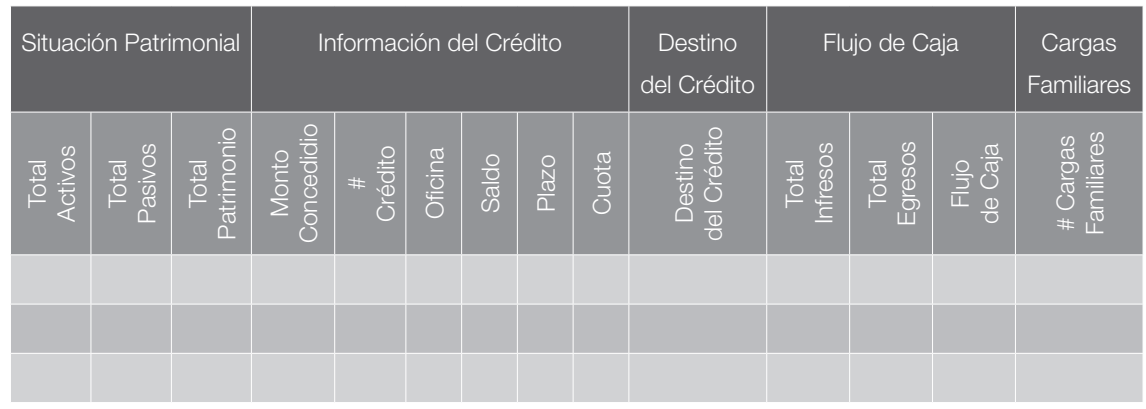

Salesiana, mediante revisión de carpetas, llamadas telefónicas y visita a los microempresarios. El documento sobre el que se apoyó la presentación de esta información corresponde a una ficha que resume los datos de los archivos de solicitud de crédito que se tienen en cada carpeta, y contiene los siguientes datos: código del socio/ nombre del microempresario, sexo, edad, estado civil, cargas familiares, nivel de instrucción, actividad económica, lugar de nacimiento, lugar de residencia, tipo de vivienda y to-

Retos I(I): 20II.

(C) 201 I, Universidad Politécnica Salesiana, Ecuador tal de activos; y fue llenada en condición ex ante (sin crédito) y ex pos (con crédito).

\section{Identificación de variables y análisis respectivo}

Las agrupamos en dos clases:

Variables intermedias: sexo, estado civil, cargas familiares, nivel de instrucción, tipo de vivienda y actividad económica. Este grupo de variables nos permite establecer el contexto de la población objetivo a través de su caracterización. 
Variables de resultados: se relacionan con el flujo de efectivo, cambios a nivel de ingresos y modificaciones en su capital de trabajo.

Como estas variables son clave para un proceso más significativo, se procedió a ubicarlas en un nivel de escalas, según los siguientes criterios:

- Disminución considerable

- Disminución moderada

- Permanece constante

- Incremento moderado

- Incremento constante

\section{Procesamiento de información, variables y modelos}

La mayoría de los créditos ha sido destinada para el consumo; el impacto real se vincula con el Capital de Trabajo, que tiene diferentes conceptos: desde el punto de vista contable, el Capital de Trabajo resulta de la diferencia entre el Activo Corriente y el Pasivo Corriente y este saldo 'sirve' para las operaciones del negocio; es decir, es la cantidad de recursos necesarios para empezar a operar un proyecto.

Con base en estos elementos se puede decir que el microcrédito ha estado relacionado con la inyección de capital para la operación de los negocios. Se determinó que los microempresarios requieren liquidez y su necesidad está enfocada en cubrir esa brecha o déficit. Esa cantidad de recursos es necesaria para el 'traba- jo normal' del negocio y en esencia permite sanear su necesidad en el corto plazo. Es por esta razón que no existe un efecto multiplicador o de incremento en otras variables matemáticas de estudio (Ingresos, $\mathrm{Pa}$ trimonio), que no tuvieron un nivel de impacto determinante. La información procesada se fundamenta en la metodología que se propuso (prueba de Wilcoxon), cuyos resultados se presentan a continuación:

El estadístico de contraste (valor Z, como se trata de una muestra grande7, la prueba se puede aproximar a una distribución normal) y la significancia asintótica nos indican que únicamente el capital de trabajo varía de un periodo a otro; es decir, del antes al después de la obtención del microcrédito.

Lo anterior nos permite afirmar que las políticas que apoyan las líneas de crédito destinadas al Capital de Trabajo son las que causan efectos en el desarrollo económico.

\section{Modelo de gestión para el microcrédito}

Este resultado evidencia que el crédito no es la solución de todos los problemas que padecen las zonas rurales con grupos económicos de ingresos bajos. Como vimos anteriormente, el crédito no se vincula con la inversión o formación de capital, sino con la cobertura de brechas de liquidez; en ese sentido, el enfoque de entregar un crédito en estas condiciones debe contextua- 
Evaluación del impacto socioeconómico del microcrédito en la zona oriental de la provincia del Azuay

\section{Cuadro 1}

\begin{tabular}{|c|c|c|c|c|}
\hline $\begin{array}{l}\text { Prueba de } \\
\text { con signo }\end{array}$ & $\begin{array}{l}\text { los rangos } \\
\text { e Wilcoxon }\end{array}$ & $\mathrm{N}$ & $\begin{array}{c}\text { Rango } \\
\text { promedio }\end{array}$ & $\begin{array}{c}\text { Suma de } \\
\text { rangos }\end{array}$ \\
\hline $\begin{array}{l}\text { Ingresos finales-Ingresos } \\
\text { iniciales }\end{array}$ & $\begin{array}{l}\text { Rangos negativos } \\
\text { Rangos positivos } \\
\text { Empates } \\
\text { Total }\end{array}$ & $\begin{array}{l}65 a \\
67 b \\
170 c \\
302\end{array}$ & $\begin{array}{l}59,19 \\
73,59\end{array}$ & $\begin{array}{l}3.847,50 \\
4.930,50\end{array}$ \\
\hline $\begin{array}{l}\text { Flujo de caja final-Flujo } \\
\text { de caja inicial }\end{array}$ & $\begin{array}{l}\text { Rangos negativos } \\
\text { Rangos positivos } \\
\text { Empates } \\
\text { Total }\end{array}$ & $\begin{array}{l}39 d \\
28 e \\
264 f \\
331\end{array}$ & $\begin{array}{l}32,24 \\
36,45\end{array}$ & $\begin{array}{l}1.257,50 \\
1.020,50\end{array}$ \\
\hline $\begin{array}{l}\text { Capital de trabajo final- } \\
\text { Capital de trabajo inicial }\end{array}$ & $\begin{array}{l}\text { Rangos negativos } \\
\text { Rangos positivos } \\
\text { Empates } \\
\text { Total }\end{array}$ & $\begin{array}{l}86 g \\
34 h \\
212 i \\
332\end{array}$ & $\begin{array}{l}56,77 \\
69,94\end{array}$ & $\begin{array}{l}4.882,00 \\
2.378,00\end{array}$ \\
\hline
\end{tabular}

a. Ingresos finales $<$ Ing resos iniciales

b. Ingresos finales $>$ Ingresos iniciales

c. Ingresos finales $=$ Ingresos iniciales

d. Flujo de caja final < Flujo de caja inicial

e. Flujo de caja final > Flujo de caja inicial

f. Flujo de caja final $=$ Flujo de caja inicial

g. Capital de trabajo final <Capital de trabajo inicial

h. Capital de trabajo final > Capital de trabajo inicial

i. Capital de trabajo final $=$ Capital de trabajo inicial

Fuente: Base de datos (muestra) Elaboración: El autor

\section{Cuadro 1}

\begin{tabular}{|llll} 
& $\begin{array}{l}\text { Ingresos finales- } \\
\text { Ingresos iniciales }\end{array}$ & $\begin{array}{l}\text { Flujo de caja final- } \\
\text { Flujo de caja inicial }\end{array}$ & $\begin{array}{l}\text { Capital de trabajo final- } \\
\text { Capital de trabajo inicial }\end{array}$ \\
\hline Z & $-1,230^{a}$ &,$- 740 \mathrm{~b}$ & $-3,279 \mathrm{~b}$ \\
Sig. asintót. (bilateral) &, 219 &, 459 &, 001 \\
\hline
\end{tabular}
a. Basado en los rangos negativos
b. Basado en los rangos positivos
c. Prueba de los rangos con signo de Wilcoxon

Fuente: Base de datos (muestra) Elaboración: El autor

lizarse a un modelo de gestión que permita establecer estrategias de intervención en el mercado objetivo. Estas estrategias se relacionan con la tasa de interés, apoyo y asesoramiento antes, durante y después de realizar las inversiones; por otro lado, es importante manejar y gestionar los montos de crédito, y las gestiones de cobranza deben ser más flexibles. 


\section{Discusión de resultados}

Por las particularidades de la información, los resultados que se obtuvieron se centraron únicamente en las modificaciones positivas o negativas de las tres variables clave que se identificaron: Flujo de Efectivo, Capital de Trabajo e Ingresos. En el transcurso del procesamiento informativo aparecieron otras categorías que permitirían profundizar la prueba de Wilcoxon en modelos de elección binaria (Modelo Logit y/o Probit) a través de la conformación de grupos de beneficiarios del microcrédito y, así, determinar las diferencias respectivas.

Un aspecto concluyente del estudio es que si bien el microcrédito es un aporte importante para mejorar las condiciones económicas de muchas familias, al favorecer positivamente el incremento del capital de trabajo, no es un factor determinante para beneficiar las economías locales. Lamentablemente, el microcrédito ha estado más vinculado al consumo que a la inversión, en el sentido de que las políticas crediticias de las instituciones financieras fomentan el consumo y no las actividades microempresariales. Por ello, las tasas de interés para créditos de consumo son más bajas que las tasas para la microempresa, lo que competitivamente desfavorece el incentivo microproductivo.

En nuestro país, no se ha realizado la cuantificación del impacto socioeconómico del microcrédito; los varios estudios sobre el impacto de las microfinanzas en Latinoamérica, Europa y Asia se han desarrollado tomando como referente al profesor Muhammad Yanus, Premio Nobel de la Paz 2006, quién planteaba una posición radical en el sentido de que el microcrédito debe erradicar la pobreza total del planeta.

En esos estudios se concluye que el microcrédito es un aporte importante para el desarrollo de los pueblos, pero que no ha logrado erradicar la pobreza en estas regiones, debido básicamente a que el microcrédito no está atado a otras actividades complementarias y paralelas que son necesarias para incidir positivamente. En este sentido, hablamos de que el acompañamiento y la asesoría en todo el ciclo del crédito resultan importantísimos. Romani Chocce (2002) habla de la cadena de impacto como "los servicios microfinancieros que llevan al cliente a modificar sus actividades microempresariales, y a aumentar o disminuir los ingresos de la microempresa. El cambio en los ingresos de la microempresa produce cambios en los ingresos del hogar, lo que lleva a una mayor o menor seguridad económica en el hogar. El nivel modificado de seguridad económica en el hogar lleva a cambios en los niveles educativos y de habilidades, y en las oportunidades económicas y sociales". Esto significa que si no consideramos el acom- 
pañamiento estructural al otorgar el microcrédito, la cadena de impacto se verá modificada o, en su defecto, será simplemente un crédito como cualquier otro que oferta el sistema financiero nacional.

\section{Notas}

[1] Factores cualitativos son descripciones de situaciones, eventos, personas y comportamientos que son observables; entre estos métodos están los estudios de caso, las entrevistas a profundidad, la observación y la investigación-acción.

[2] La zona oriental de la provincia del Azuay (cuenca del río Paute) a diferencia de la cuenca del Jubones, aparte del impacto negativo que generó la crisis económica de 1999, se caracteriza por el profundo retroceso socioeconómico generado por el Desastre de la Josefina en el año 1993, cuando un deslizamiento taponó el cauce del río Paute, y la inundación y su desfogue destruyeron viviendas y cultivos aguas arriba y aguas abajo del deslave.

[3] La ficha de solicitud de crédito es un formulario que cada banco, mutualista o cooperativa dispone para levantar la información de sus clientes: personal, económica, financiera, capacidad de pago, garantías, etcétera, que sirve para evaluar las condiciones en que se encuentra el solicitante. En la actualidad, en nuestro país, este documento es estándar, homogéneo y regulado por la Superintendencia de Bancos y Seguros.

[4] La información que se registró es considerada confidencial, por lo que se realizaron muchas sesiones de trabajo con los directivos de las instituciones financieras para explicar la lógica, los procedimientos y los resultados posibles del proceso investigativo.

[5] Es una prueba no paramétrica que sirve para comparar la media de dos muestras relacio- nadas y determinar si existen diferencia entre ellas.

[6] O de libre distribución, cuando no se puede usar la distribución normal, los test no paramétricos permiten hacer uso de algunas herramientas que no necesitan que la variable siga una determinada distribución.

[7] Se considera una muestra grande cuando supera las veinte observaciones.

\section{Bibliografía}

Textos

Gujarati, Damodar, Econometría, Cuarta Edición, Editorial Mc Graw Hill, 2004.

Shmidt, Stephen J., Econometría, Primera Edición, Editorial Mc Graw Hill, 2005.

Pindyck, Robert y Rubienfeld, Daniel, Econometría, modelos y pronósticos, Primera Edición, Editorial Mc Graw Hill, 2001.

Stiglitz, Joseph, Microeconomía, Segunda Edición, Editorial Ariel S.A, 1998.

Mochon, Francisco, Principios de Economía, Tercera Edición, Editorial Mc Graw Hill, 2006.

BID, La Microempresa en Ecuador, perspectivas, desafíos y línea de apoyo, publicación del Banco Interamericano de Desarrollo, 2006.

Aldunate, Eduardo, Bases metodológicas para la programación y administración eficiente de la Inversión Pública, ILPES, 2005.

Contreras, Eduardo, Evaluación social de inversiones públicas, enfoques alternativos y su aplicabilidad para América Latina, ILPES, 2005.

Navarro, Hugo, El uso de indicadores socioeconómicos en la formulación y evaluación de proyectos sociales, ILPES, 2005.

Navarro, Hugo, Manual para la evaluación de impacto de proyectos y programas de lucha contra la pobreza, ILPES, 2005.

Agradecemos a la Cooperativa de Ahorro y Crédito Jardín Azuayo por su colaboración en este proyecto.

envío 28 de enero de 2011 - aprobación 8 de marzo de 2011

Retos I(I): 20II.

(C) 201I, Universidad Politécnica Salesiana, Ecuador 